\title{
Forecasting the COVID-19 transmission in Italy based on the minimum spanning tree of dynamic region network
}

\author{
Min Dong ${ }^{1}$, Xuhang Zhang ${ }^{1}$, Kun Yang ${ }^{1}$, Rui Liu ${ }^{\text {Corresp., } 2,3}$, Pei Chen ${ }^{\text {Corresp. } 2}$ \\ ${ }^{1}$ School of Computer Science and Engineering, South China University of Technology, Guangzhou, China \\ 2 School of Mathematics, South China University of Technology, Guangzhou, China \\ 3 Pazhou Lab, Guangzhou, Guangdong, China \\ Corresponding Authors: Rui Liu, Pei Chen \\ Email address: scliurui@scut.edu.cn, chenpei@scut.edu.cn
}

Background: Italy surpassed 1.5 million confirmed Coronavirus Disease 2019 (COVID-19) infections on November 26, as its death toll rose rapidly in the second wave of COVID-19 outbreak which is a heavy burden on hospitals. Therefore, it is necessary to forecast and early warn the potential outbreak of COVID-19 in the future, which facilitates the timely implementation of appropriate control measures. However, real-time prediction of COVID-19 transmission and outbreaks is usually challenging because of its complexity intertwining both biological systems and social systems.

Methods: By mining the dynamical information from region networks and the short-term time series data, we developed a data-driven model, the minimum-spanning-tree-based dynamical network marker (MST-DNM), to quantitatively analyze and monitor the dynamical process of COVID-19 spreading. Specifically, we collected the historical information of daily cases caused by COVID-19 infection in Italy from February 24, 2020 to November 28, 2020. When applied to the region network of Italy, the MSTDNM model has the ability to monitor the whole process of COVID-19 transmission and successfully identify the early-warning signals. And the interpretability and practical significance of our model are explained in detail in this study.

Results: The study on the dynamical changes of Italian region networks reveals the dynamic of COVID-19 transmission at the network level. It is noteworthy that the driving force of MST-DNM only relies on small samples rather than years of time series data. Therefore, it is of great potential in public surveillance for emerging infectious diseases. 
1 Forecasting the COVID-19 transmission in Italy based

2 on the minimum spanning tree of dynamic region

3 network

4

5

6

7

8

Min Dong ${ }^{1}$, Xuhang Zhang ${ }^{1}$, Kun Yang ${ }^{1}$, Rui Liu ${ }^{2,3^{*}}$, Pei Chen ${ }^{2 *}$

${ }^{1}$ School of Computer Science and Engineering, South China University of Technology, Guangzhou, Guangdong, China

${ }^{2}$ School of Mathematics, South China University of Technology, Guangzhou, Guangdong, China

${ }^{3}$ Pazhou Lab, Guangzhou, Guangdong, China.

Corresponding Author:

Rui Liu ${ }^{2,3}$, Pei Chen ${ }^{2}$

Wushan Campus, South China University of Technology, Guangzhou, Guangdong, 510640, China

Email address: R.L.scliurui@scut.edu.cn and P.C. $\underline{\text { chenpei@scut.edu.cn }}$

\section{Abstract}

Background: Italy surpassed 1.5 million confirmed Coronavirus Disease 2019 (COVID19) infections on November 26, as its death toll rose rapidly in the second wave of COVID-19 outbreak which is a heavy burden on hospitals. Therefore, it is necessary to forecast and early warn the potential outbreak of COVID-19 in the future, which facilitates the timely implementation of appropriate control measures. However, real-time prediction of COVID-19 transmission and outbreaks is usually challenging because of its complexity intertwining both biological systems and social systems.

Methods: By mining the dynamical information from region networks and the short-term time series data, we developed a data-driven model, the minimum-spanning-tree-based dynamical network marker (MST-DNM), to quantitatively analyze and monitor the dynamical process of COVID-19 spreading. Specifically, we collected the historical information of daily cases caused by COVID-19 infection in Italy from February 24, 2020 to November 28, 2020. When applied to the region network of Italy, the MST-DNM model has the ability to monitor the whole process of COVID-19 transmission and successfully identify the early-warning signals. And the interpretability and practical significance of our model are explained in detail in this study.

Results: The study on the dynamical changes of Italian region networks reveals the dynamic of COVID-19 transmission at the network level. It is noteworthy that the driving force 
38

of MST-DNM only relies on small samples rather than years of time series data. Therefore, it is of great potential in public surveillance for emerging infectious diseases.

\section{Introduction}

The world is currently witnessing a major and devastating pandemic with substantial mortality and morbidity-Coronavirus Disease 2019 (COVID-19) [1]. It was declared by the World Health Organization (WHO) as a public health emergency of international concern in January 2020 [2][3]. As of November 28, 2020, about 60 million cases and 1.45 million deaths were confirmed globally. Italy is one of the most affected countries. In about two months, i.e., from midFebruary 2020 to mid-April 2020, it has been one of the main epicenters of the COVID-19 pandemic when the epidemic reached its first peak. Then, the epidemic curve gradually decreased until early October 2020, after which the spread of infection accelerated again until today [4]. As shown in Fig. 1A, a new and more severe epidemic is sweeping Italy. As of November 28, Italy had suffered 54,363 deaths and 1,564,532 cases [5]. Therefore, there is an urgent need for an effective and low-cost model to build an epidemic surveillance system to help countries severely affected by the epidemic like Italy to warn of a new wave of COVID-19 outbreaks in the future.

The COVID-19 pandemic has sparked an intense debate about the factors underlying the dynamics of the outbreak [6][7]. Meanwhile, the study of mathematical models of epidemiology is helpful to understand the dynamics of epidemics, being an important tool to evaluate the potential effects of preventive and controlled measures, especially when their characteristics are still unclear [8][9][10].Under such circumstances, by exploring the dynamical information from region networks and time-series data, we employed a combined model, the minimum-spanningtree-based dynamical network marker (MST-DNM) [11], to quantitatively describe the dynamics of COVID-19 transmission and thus identify the early-warning signal of a new wave of COVID19 pandemic in Italy. This is the first time that this model has been applied to the study of the emerging infectious disease like COVID-19. This model is improved from our recently proposed concept, the so-called dynamical network biomarker (DNB), which determines the critical state of complex diseases by analyzing the dynamics of driven biomolecules (i.e., a group of genes and proteins that are the leading factors to the critical state transition) [12]. The DNB based methods have been applied to a number of biological progresses and obtained remarkable results, including identifying the critical points of cell fate decision [13] and cellular differentiation [14], detecting the critical period during various biological processes [15][16][17][18], and predicting the warning signals of influenza outbreaks [11][19][20].

By extracting the minimum spanning tree from the dynamic region network, we could typically describe the dynamics of the spread of the infectious diseases among regions. Specifically, we collected the historical information of daily cases by COVID-19 infection in Italy from February 24, 2020 to November 28, 2020. When applied to the region networks constructed based on geographical location and traffic conditions, the MST-DNM model has the ability to monitor the whole process of epidemic spread in Italy, and successfully identify the 
77 early-warning signals about two weeks in advance. It is worth noting that in the previous

78 research, the key role of the minimum spanning tree in this model has been described in detail

79 [11], which can avoid issuing wrong warning signals due to the appearance of local abnormal

80 correlation. Therefore, we pay more attention to the interpretability and practical significance of

81 the MST-DNM model in our study, as detailed in Methods and Results section. Consequently,

82 our model is quite suitable for predicting the potential outbreak of COVID-19 in Italy with the

83 characteristics of nonlinearity time series and small sample size based on Italian region network,

84 which may help to develop new control strategies for COVID-19 before its new wave of

85 outbreaks.

86

87

88

89

90

91

92

93

94

95

96

97

98

99

100

101

102

103

104

105

106

107

108

109

110

111

112

113

114

115

\section{Materials \& Methods}

\section{Theoretical background}

The spread of infectious diseases in a region is described as the dynamic evolution of a nonlinear system, while the outbreak of COVID-19 is regarded as a qualitative state transition of a dynamic system [11]. From the perspective of dynamic modelling, the stage before the COVID-19 outbreak is regarded as a pre-outbreak stage, immediately after which the system undergoes a critical transition. Then the dynamical process of an epidemic system can be roughly modelled as three stages similar to the dynamics of disease progression [12]: (i) the normal stage, which is a stable stage with high resilience; (ii) the pre-outbreak stage, which is dynamically unstable. At this stage, the epidemic is still controllable through appropriate measures; and (iii) the outbreak stage, which is an uncontrolled stage with high elastic dynamic features. As shown in Fig. 1B, when the system transits from the normal stage to the pre-outbreak stage, the region network changes significantly and the indicator of our model rises sharply. Different from the traditional detection of the outbreak stage, our model could determine the pre-outbreak stage which generally has no obvious abnormalities but with high potential of state transition into a severe and irreversible stage.

\section{Data collection}

Data about the COVID-19 pandemic can be obtained from the GitHub repository managed by Johns Hopkins University for modeling [5], which contains publicly available data from multiple sources. The open dataset of Italy we use is available on the website COVID-19 in Italy at: https://github.com/pcm-dpc/COVID-19. This database has been created and managed by the Italian Civil Protection Department, which is updated and integrated daily [21].

\section{Algorithm applied in Italy}

The MST-DNM model is illustrated in Fig. 2 and the detailed process of our model applied in Italy is described in the following four steps.

\section{i. Modeling and mapping}

It is noted that our model is applied to the region network to monitor the COVID-19 transmission and outbreak in Italy. Therefore, it is necessary to construct the regional network based on the Italian regions' geographic distribution and their adjacent information at first. The adjacent information is shown in Table S1 of Supplementary Information. In the network, each 
116 node represents a region or an autonomous province, while each edge represents the adjacent 117 relation between two regions. Then a $21 * 279$ data matrix formed by the records of COVID-19 118 daily confirmed cases is mapped to the network. The region network model of Italy is presented 119 as in Fig. 3.

\section{ii. Weighting and extracting}

121

122

The region network in the first step can be represented as an undirected graph $G=(V, E)$, which contains a collection $V=\left\{v_{i}\right\}_{i=1}^{M}$ composed of $M$ vertexes and a collection $E=\left\{e_{j}\right\}_{j=1}^{N}$

123

124

125

126

127

128

129

130

131

132

133

134

where

$$
\Delta S D_{t}(i, j)=\overline{S D_{t}\left(S_{t}^{v_{i}}\right)+S D_{t}\left(S_{t}^{v_{j}}\right)}-\overline{S D_{t-1}\left(S_{t-1}^{v_{i}}\right)+S D_{t-1}\left(S_{t-1}^{v_{j}}\right)} \mid
$$

135 is the differential standard deviation of the nodes $v_{i}, v_{j}$ on day $t$ and $t-1$, and $S D_{t}\left(S_{t}^{v_{i}}\right)$ and $S D_{t}$ 136 $\left(s_{t}^{v_{j}}\right)$ represent the standard deviation of the time series data of the two vertices $v_{i}, v_{j}$.

$$
\Delta P C C_{t}(i, j)=\left|P C C_{t}\left(S_{t}^{v_{i}}, S_{t}^{v_{j}}\right)\right|-\left|P C C_{t-1}\left(S_{t-1}{ }^{v_{i}}, S_{t-1}{ }^{v_{j}}\right)\right|
$$

137

138

139

140

141

142

143

144

145

146

is the differential Pearson's correlation coefficient between the two vertices $v_{i}, v_{j}$ of the edge $e^{k}$, where $\operatorname{PCC}_{t}\left(S_{t}^{v_{i}}, S_{t}^{v_{j}}\right)$ and $P C C_{t-1}\left(S_{t-1}{ }^{v_{i}}, S_{t-1}{ }^{v_{j}}\right)$ represent the Pearson's correlation coefficient between the two vertices $v_{i}, v_{j}$ on day $t$ and $t-1$ respectively.

Based on the above work, an undirected and edge-weighted network that changes dynamically over time is obtained. The next step is to extract the minimum spanning tree from the dynamic region network at each moment. In detail, when Italy is on the $t$ day of the COVID19 pandemic, we could extract the minimum spanning tree $M_{S} T_{t}=(V, E)$ to better describe the evolution of Italian regional network with the change of daily cases. In this work, a classical minimum spanning tree algorithm, the Prim's algorithm, is applied to the differential weighted network $G_{t}$ at a specific time $t$ to obtain its minimum spanning tree $M S T_{t}$. 
147

148

149

150

151

152

153

154

155

156

157

158

159

160

161

162

163

164

165

166

167

168

169

170

171

172

173

174

175

176

177

178

179

180

181

182

183

\section{iii. Calculating early warning indicators $I_{t}$}

Then, the MST-DNM indicator $I_{t}$ can be obtained by calculating the weight sum of the minimum spanning tree. $I_{t}=\sum_{i=1}^{K} W_{t}^{i}$, where $W_{t}^{i}$ represents the weight of the edge $e_{i}$ of the minimum spanning tree $M S T_{t}$ at time $t$ and $K$ is the total number of edges of the minimum spanning tree $M S T_{t}$. The specific algorithm flow is shown in Algorithm 1.

According to DNB theory, during the critical stage, there are two cases for the minimum spanning tree $M S T_{t}$ at time point $t$ :

- In the $M S T_{t}$, all of the nodes are DNB members;

- In the $M S T_{t}$, DNB and non-DNB members both exist.

For the above two cases respectively, the statistical indicator $I_{t}$ has significant changes as presented in Table 1. Obviously, the $M S T_{t}$ based on the indicator $I_{t}$ and the edges' weight $W_{t}$ has the ability to monitor the dynamical process of COVID-19 spread between regions and issue a warning signal timely.

\section{iv. Identifying early-warning points}

In previous studies, machine learning methods, i.e., logistic regression [11], have been applied to identify the appearance of critical points based on years of high-dimensional data. However, for COVID-19 which originated at the beginning of 2020, the time series data obtained is of quite small scale, which is difficult for machine learning algorithm to learn the appropriate parameters and features. Therefore, the fold-change threshold, an index of volatility, is used to detect the early-warning signal. Specifically, a 2-fold change threshold is applied to identify the significant changes of the indicator $I_{t}$ in our study.

\section{The significance of indicator $I_{t}$}

The transmission of COVID-19 is a complicated dynamic system, which contains many biomedical and social factors. Due to the massive number of influencing factors, it is difficult to describe the transport dynamics in high-dimensional space mathematically. The sharp or qualitative transition of regional network from the normal state to the outbreak state corresponds to the bifurcation point in dynamic system theory [22]. According to this theory, if the system approaches the critical point, it will eventually be confined to one-dimensional or twodimensional space, where the dynamic system can be represented in quite simple forms. This is the theoretical basis for developing a general indicator that can describe the dynamics of COVID-19 transmission.

It's clear from the above statement that the meaning of the variables in Formula (1) is as follows: (i) $\left|\Delta S D_{t}(i, j)\right|$ can describe the differential fluctuation of cases growth in two adjacent regions compared with the previous time point. (ii) $\left|\triangle P C C_{t}(i, j)\right|$ can describe the difference of the COVID-19 interaction between two adjacent regions compared with the previous time point. Apparently, attention should be paid to the edge with larger weight. Because it means that the regions associated with this edge not only worsen their own epidemic situation, but also have a 
184 great impact on the surrounding regions. Therefore, it's obvious that the indicator $I_{t}$, the weight

185 sum $W_{t}^{k}$ of all edges in $M S T_{t}$, has the ability to observe the change of a group of weighted 186 differential networks.

\section{Results}

188 Early warning of COVID-19 outbreaks in Italy

189 We collect the historical data of daily cases infected by COVID-19 from February 24, 2020 to

190 November 28, 2020 in Italy. The outbreak points of COVID-19 are defined as the peak of the

191 daily cases.

192 Provided as in Fig. 4, the early-warning signals are identified through the MST-DNM 193 model for each outbreak of COVID-19. For the first wave of COVID-19 outbreak from mid194 February to mid-April, the early-warning signal was issued on March 6, which is about 15 days 195 ahead of the outbreak point. This means that our model has successfully played an early warning 196 role.

On 9 March 2020, the Italian prime minister Mr. G Conte announced the implementation of placing the country into lockdown to restrict the movement of people, thus reducing the possibility of human to human infection [23][24]. Since the last week of March, the statistics have become consciously optimistic, and the number of daily cases has been stabilizing. For the

201

202 second wave of epidemic since early October, later developing into a larger outbreak, the indicator $I_{t}$ was sensitive and significantly increased about 10 days before the actual number of

203 confirmed cases skyrockets. In addition, the indicator showed a continuous downward trend with wave type after November 5, which means that the number of daily cases in Italy has initially peaked. The successful prediction of each wave of COVID-19 outbreaks in Italy demonstrates the robustness and effectiveness of the MST-DNM model in detecting real-time warning signals

207 for emerging infectious diseases.

208 The dynamics of COVID-19 transmission in Italy

209 Dynamic monitoring map

210 To better illustrate the MST-DNM model's principle, we introduce the dynamic evolution of the

211 COVID-19 transmission network in Italy. As shown in Fig. 5, the daily number of newly

212 confirmed cases with MinMaxScaler in each region is mapped to each node and the correlation

213 between two vertices of an edge is mapped to the thickness of the edge in the tree network. The

214 specific method of MinMaxScaler is to subtract the minimum value of the feature from the

215 processed value and divide it by the feature range, which is the difference between the original

216 maximum and the original minimum. MinMaxScaler can keep the shape of the original data

217 distribution of each region, and make the coloring of each node unaffected by other regions. It is

218 clear that the edges became thicker before the nodes turned darker on October 20, which

219 indicated our model identified the early-warning signal in the pre-outbreak stage when the actual

220 number of confirmed cases did not increase significantly. After that, i.e., on October 26, the 
221 edges continued to become thicker, which meant that the epidemic might continue to worsen in 222 Italy.

\section{Warning function of MST-DNM in Italy}

224 As of November 28, the five regions with the highest cumulative number of confirmed cases in

225 Italy are Lombardy, Piedmont, Campania, Veneto and Emilia-Romagna, which correspond to

226 Nodes 10, 13, 5, 21 and 6 in Fig. 6A, respectively. Among them, Lombardy region is considered 227 as the epicenter of COVID-19 outbreak in Italy [25][26].

228 As shown in Fig. 6A, the dynamic region network is divided into two local networks 229 according to the thickness of the edges, which are centered on Node 10 (Lombardy) and Node 5

230 (Campania) respectively. It's obvious that our model has successfully warned two high-risk

231 regions. In fact, a large number of new cases have been confirmed in these regions over the next 232 month as shown in Fig. 6B. In addition, the nodes sandwiched between two local networks, i.e., 233 Node 17 (Tuscany) and Node 19 (Umbria), should also be focused on. It turns out that from 234 October 26 to November 28, the growth rate of new case in Tuscany and Umbria exceeded $235200 \%$.

236 As described in Table 2, the thicker edges in two local networks on October 26, such as $e$ $237\left(v_{10}, v_{21}\right), e\left(v_{6}, v_{21}\right), e\left(v_{13}, v_{20}\right), e\left(v_{5}, v_{8}\right)$ and $e\left(v_{12}, v_{14}\right)$, should be focused on. As of 238 November 28, the number of confirmed cases in the regions, like Lombardy, Campania, Lazio 239 and Piedmont, corresponding to the nodes associated with these edges has a high growth rate and 240 a large number of new cases. This also verify the early warning function of MST-DNM, which 241 not only measures its own epidemic situation, but also reflects the regional impact on the 242 surrounding areas.

\section{Application of MST-DNM in northern Italy}

244 As shown in Fig. 6B, several areas in northern Italy, such as Lombardy, Veneto, etc., are the 245 most severely affected by COVID-19. Our model can be applied not only to the entire country of 246 Italy, but also to an area. In order to verify the effectiveness of our model, it has also been 247 applied to identify the early-warning signals of COVID-19 outbreaks in northern Italy. The 248 results are presented in Fig. S2-S3 of Supplementary Information.

\section{Performance comparison}

250 The machine learning algorithms are also used to forecast the COVID-19 epidemic 251 situation[27][28][29]. Regarding the identification of early warning signals of COVID-19 252 outbreaks as a binary classification problem, we compare the performance of our combined 253 model with the support vector machine (SVM). The AUC of MST-DNM is 0.9318, while that of 254 SVM is 0.9076. It's clear that the performance of a system based on MST-DNM is better than 255 that based on SVM when only the data of daily confirmed cases is provided. In addition, the 256 SVM model issue an early warning signal on September 28, 2020, which is too early to be of 257 practical significance for the second wave outbreak; and our adaptive model can actually issue an 258 early warning signal about 10 days before the actual number of confirmed cases skyrockets. 259 Actually, compared with traditional machine learning algorithms, the MST-DNM model has the 260 following internal strengths. First of all, it is a model-free approach without any training and 
261

262

263

264

265

266

267

268

269

270

271

272

273

274

275

276

277

278

279

280

281

282

283

284

285

286

287

288

289

290

291

292

293

294

295

296

297

298

testing processes. There is no feature selection in MST-DNM strategy, which solely depends on the statistical conditions of our model. Second, it's noted that there is no limitation of the data sample size for our approach, which means that our model could achieve a good performance with only small sample data. Therefore, it can be applied to describe and monitor the emerging infectious diseases like COVID-19. In addition, our combined model is capable to describe the dynamic process of the spread of COVID-19 through the minimum spanning tree of dynamic region networks.

\section{Discussion}

A new wave of COVID-19 epidemic is sweeping the world. On November 26, more than 1.5 million people were diagnosed with COVID-19 infection in Italy, and the death toll rose rapidly in the second wave of COVID-19 epidemic, bringing a heavy burden to hospitals. In order to prevent a new wave of COVID-19 pandemic or at least reduce the magnitude of COVID-19 outbreaks, it is essential to build a surveillance system that relies entirely on reliable and available information, such as the number of daily cases.

Specifically, unlike the critical transformation analysis based on DNB of complex diseases with genomic datasets, the DNB method has been improved and applied to the macro regional networks. The successful application in Italy shows that the MST-DNM is a model-free method with data-driven characteristics and has great potential in actual real-time monitoring for emerging infectious diseases. Moreover, this is the first time that the improved method based on DNB has been applied to predict the outbreak of COVID-19. Unlike previous studies that used the DNB based methods to predict influenza outbreaks[11][19][20], our study is based on small time series samples rather than years of time series data. Therefore, it could be employed to describe and monitor emerging infectious diseases like COVID-19. In addition, this paper introduces the practical significance and early warning function of the MST-DNM model in detail. It is believed that this is an important step from theory to practice. It should be noted that the MST-DNM model in our work is completely based on the records of confirmed cases per day, and has achieved satisfactory performance. Given more information on the spread of the COVID-19 epidemic, the monitoring model is expected to reliably forecast the transmission and outbreak of COVID-19 in terms of sensitivity and accuracy.

Although the proposed model have achieved good results, there are some limitations of the project:

- The MST-DNM model in our work is completely based on the records of daily cases. If we could get data on the number of people tested in a region, we could measure the epidemic situation in this region more accurately. This may be the direction of model improvement in the future.

- As for the recognition of early warning signals, we can take into consideration any alternative to that criterion and the effect that a different choice could have on the prediction results in the future work. 

future work could involve to exam the proposed model on other regions or countries.

\section{Conclusions}

302 In this study, we developed a combined model with dynamic network marker and minimum 303 spanning tree solely based on the daily cases to describe and forecast the COVID-19 outbreaks in 304 Italy. In order to put theory into practice, we also explain the significance and warning function 305 of the model indicators in detail. By extracting the minimum spanning tree from the dynamic 306 region network, the model can effectively identify the early-warning signals with an average of 2-week window lead prior to the catastrophic transition into COVID-19 outbreaks in Italy.

308

309

310

311

312

313

314

315

316

317

318

319

320

321

322

323

324

325

326

327

328

329

330 Through the study of the network dynamics in Italy, this paper reveals the spread of COVID-19 on the network level. It is noteworthy that the driving force of MST-DNM only relies on small samples, rather than multi-year data. Therefore, it has great potential to monitor emerging infectious diseases timely.

\section{Acknowledgements}

We would like to thank Professor Sheng Bi and Mr. Yingqi Chen for productive discussions.

\section{Declarations}

\section{Ethics approval and consent to participate}

Not applicable.

\section{Consent for publication}

Not applicable.

\section{Competing interests}

The authors declare that they have no competing interests.

\section{Funding}

This work was supported by National Natural Science Foundation of China (Nos. 61703168, 11771152, 12026608, 11901203, 11971176), Guangdong Basic and Applied Basic Research Foundation (2019B151502062), China Postdoctoral Science Foundation funded project (No. 2019M662895, 2020T130212) and the Fundamental Research Funds for the Central Universities (2019MS111).

\section{Author's Contributions}

$\mathrm{MD}, \mathrm{XHZ}, \mathrm{RL}$ and PC designed the research; MD, XHZ and KY performed the experiments and created the figures; XHZ and PC wrote and edited the manuscript. RL and PC provided direction for the project and its goals. All authors read and approved the final manuscript. 


\section{References}

332 [1] Mohanty S K, Satapathy A, Naidu M M, et al. Severe acute respiratory syndrome

333

334

335

336

337

338

339

340

341

342

343

344

345

346

347

348

349

350

351

352

353

354

355

356

357

358

359

360

361

362

363

364

365

366

367

368

369

370 coronavirus-2 (SARS-CoV-2) and coronavirus disease 19 (COVID-19)-anatomic pathology perspective on current knowledge. Diagnostic pathology, 2020, 15(1): 1-17.

[2] Team EE, Eurosurveillance editorial team, Eurosurveillance Editorial Team. Note from the editors: World Health Organization declares novel coronavirus (2019-nCoV) sixth public health emergency of international concern. Euro surveillance : bulletin européen sur les maladies transmissibles. 2020;25(5):2-3.

[3] Lai C, Shih T, Ko W, Tang H, Hsueh P. Severe acute respiratory syndrome coronavirus 2 (SARS-CoV-2) and coronavirus disease-2019 (COVID-19): The epidemic and the challenges. International journal of antimicrobial agents. 2020;55(3):105924-.

[4] Perone G. Comparison of ARIMA, ETS, NNAR and hybrid models to forecast the second wave of COVID-19 hospitalizations in Italy. arXiv preprint arXiv:2010.11617, 2020.

[5] Dong E, Du H, Gardner L. An interactive web-based dashboard to track COVID-19 in real time. The Lancet infectious diseases. 2020;20(5):533-4.

[6] Coelho M T P, Rodrigues J F M, Medina A M, et al. Global expansion of COVID-19 pandemic is driven by population size and airport connections. PeerJ, 2020, 8: e9708.

[7] Pequeno P, Mendel B, Rosa C, et al. Air transportation, population density and temperature predict the spread of COVID-19 in Brazil. PeerJ, 2020, 8: e9322.

[8] Chatterjee K, Chatterjee K, Kumar A, Shankar S. Healthcare impact of COVID-19 epidemic in India: A stochastic mathematical model. Medical journal. Armed Forces India. 2020;76(2):147-55.

[9] Khan T, Ullah Z, Ali N, Zaman G. Modeling and control of the hepatitis B virus spreading using an epidemic model. Chaos, solitons and fractals. 2019;124:1-9.

[10] Cheng X, Han Z, Abba B, Wang H. Regional infectious risk prediction of COVID-19 based on geo-spatial data. PeerJ (San Francisco, CA). 2020.

[11] Yang K, Xie J, Xie R, Pan Y, Liu R, Chen P. Real-Time Forecast of Influenza Outbreak Using Dynamic Network Marker Based on Minimum Spanning Tree. BioMed research international. 2020;2020:7351398-.

[12] Chen L, Liu R, Liu Z, Li M, Aihara K. Detecting early-warning signals for sudden deterioration of complex diseases by dynamical network biomarkers. Scientific Reports. 2012;2(1):342.

[13] Mojtahedi M, Skupin A, Zhou J, Castaño IG, Leong-Quong RYY, Chang H, et al. Cell Fate Decision as High-Dimensional Critical State Transition. PLoS Biology. 2016;14(12):e2000640.

[14] Richard A, Boullu L, Herbach U, Bonnafoux A, Morin V, Vallin E, et al. Single-Cell-Based Analysis Highlights a Surge in Cell-to-Cell Molecular Variability Preceding Irreversible Commitment in a Differentiation Process. PLoS Biology. 2016;14(12):e1002585.

[15] Liu R, Yu X, Liu X, Xu D, Aihara K, Chen L. Identifying critical transitions of complex diseases based on a single sample. Bioinformatics. 2014;30(11):1579-86. 
371 [16] Liu R, Wang J, Ukai M, Sewon K, Chen P, Suzuki Y, et al. Hunt for the tipping point during

372

373

374

375

376

377

378

379

380

381

382

383

384

385

386

387

388

389

390

391

392

393

394

395

396

397

398

399

400

401

402 molecular cell biology. 2019;11(8):649-64.

[17] Chen P, Liu R, Chen L, Aihara K. Identifying critical differentiation state of MCF-7 cells for breast cancer by dynamical network biomarkers. Frontiers in Genetics. 2015;6:252.

[18] Chen P, Li Y, Liu X, Liu R, Chen L. Detecting the tipping points in a three-state model of complex diseases by temporal differential networks. Journal of Translational Medicine. 2017;15(1):217-15.

[19] Chen P, Chen E, Chen L, Zhou XJ, Liu R. Detecting early-warning signals of influenza outbreak based on dynamic network marker. Journal of Cellular and Molecular Medicine. 2019;23(1):395-404.

[20] Chen Y, Yang K, Xie J, Xie R, Liu Z, Liu R, et al. Detecting the outbreak of influenza based on the shortest path of dynamic city network. PeerJ (San Francisco, CA). 2020;8:e9432-.

[21] Morettini M, Sbrollini A, Marcantoni I, et al. COVID-19 in Italy: Dataset of the Italian Civil Protection Department. Data in Brief, 2020: 105526.

[22] Gilmore R. Catastrophe theory for scientists and engineers. Courier Corporation; 1993.

[23] Chintalapudi N, Battineni G, Amenta F. COVID-19 virus outbreak forecasting of registered and recovered cases after sixty day lockdown in Italy: A data driven model approach.

Journal of microbiology, immunology and infection. 2020;53(3):396-403.

[24] Remuzzi A, Remuzzi G. COVID-19 and Italy: what next?. The Lancet, 2020.

[25] Grasselli G, Pesenti A, Cecconi M. Critical Care Utilization for the COVID-19 Outbreak in Lombardy, Italy: Early Experience and Forecast during an Emergency Response. JAMA : the journal of the American Medical Association. 2020;323(16):1545-6.

[26] Tuite AR, Ng V, Rees E, Fisman D. Estimation of COVID-19 outbreak size in Italy. The Lancet infectious diseases. 2020;20(5):537-.

[27] Parhusip H A. Study on COVID-19 in the World and Indonesia Using Regression Model of SVM, Bayesian Ridge and Gaussian. JURNAL ILMIAH SAINS, 2020, 20(2): 49-57.

[28] Singh S, Parmar K S, Makkhan S J S, et al. Study of ARIMA and least square support vector machine (LS-SVM) models for the prediction of SARS-CoV-2 confirmed cases in the most affected countries. Chaos, Solitons \& Fractals, 2020, 139: 110086..

[29] Parbat D, Chakraborty M. A python based support vector regression model for prediction of COVID19 cases in India. Chaos, Solitons \& Fractals, 2020, 138: 109942. 
Figure 1

Schematic diagram of COVID-19 pandemic in Italy and prediction principle.

(A) From mid-February 2020 to mid-April 2020, the epidemic in Italy reached its first peak. Since then, the epidemic curve gradually declined until early October, and then the spread of COVID-19 infection accelerated again until today. A new wave of COVID-19 outbreaks is affecting Italy. (B) According to the MST-DNM model, the process of COVID-19 outbreaks is divided into three stages, including the normal stage, the pre-outbreak stage and the outbreak stage. The sudden increase in the MST-DNM indicator indicates a transition from the normal stage to the pre-outbreak stage, i.e., the critical point before the upcoming outbreak of COVID-19 that results in an increase in daily cases.

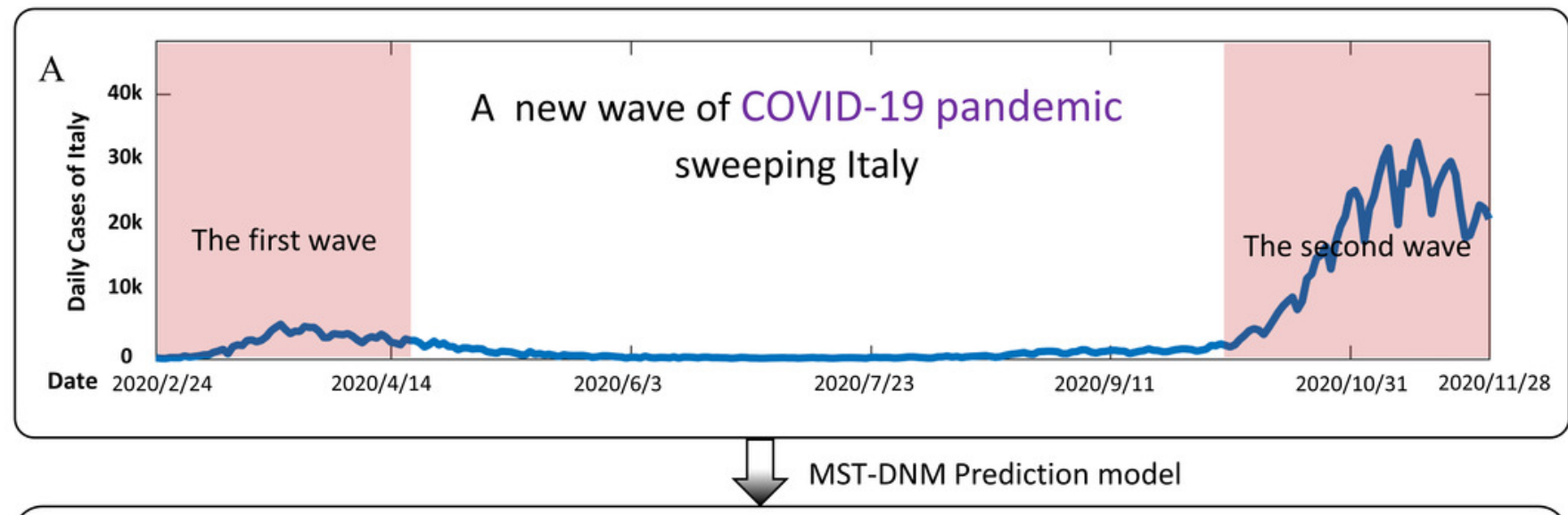

B An early warning signal exists before the outbreak of COVID-19

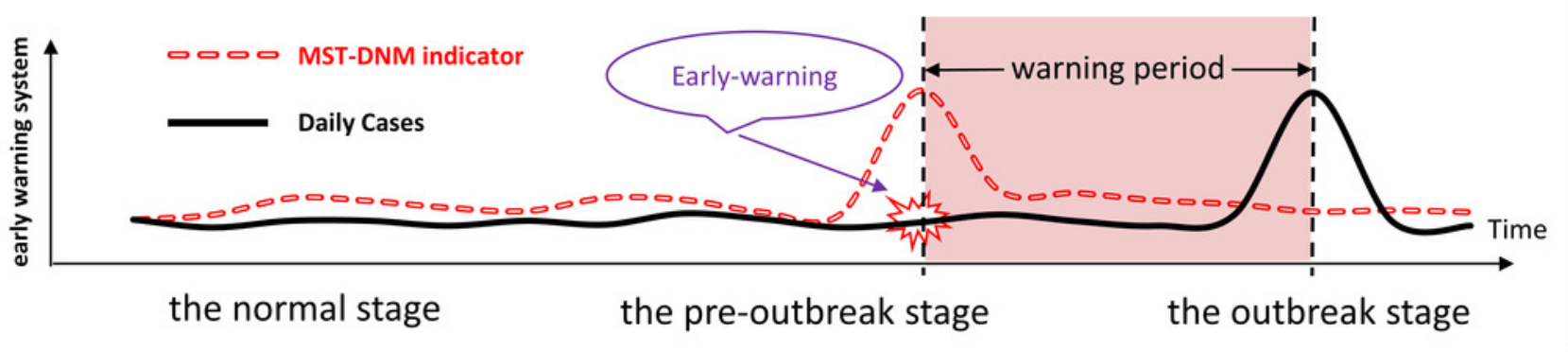




\section{Figure 2}

The flow chart of algorithm application in Italy.

The flow chart above shows how the algorithm works in Italy based on region networks and minimum spanning tree. The model is driven by time series and dynamic region networks over time. Regarding a point $T=t$ as a candidate tipping point, MST-DNM indications can be calculated based on weighted region networks. If the indication increases significantly, the candidate $T=t$ is determined as the identified tipping point, and the algorithm ends. Otherwise, if there is no significant change, then $T=t$ is classified as a time point belonging to the normal stage, and the algorithm continues with $T=t+1$ being a new candidate tipping point. 


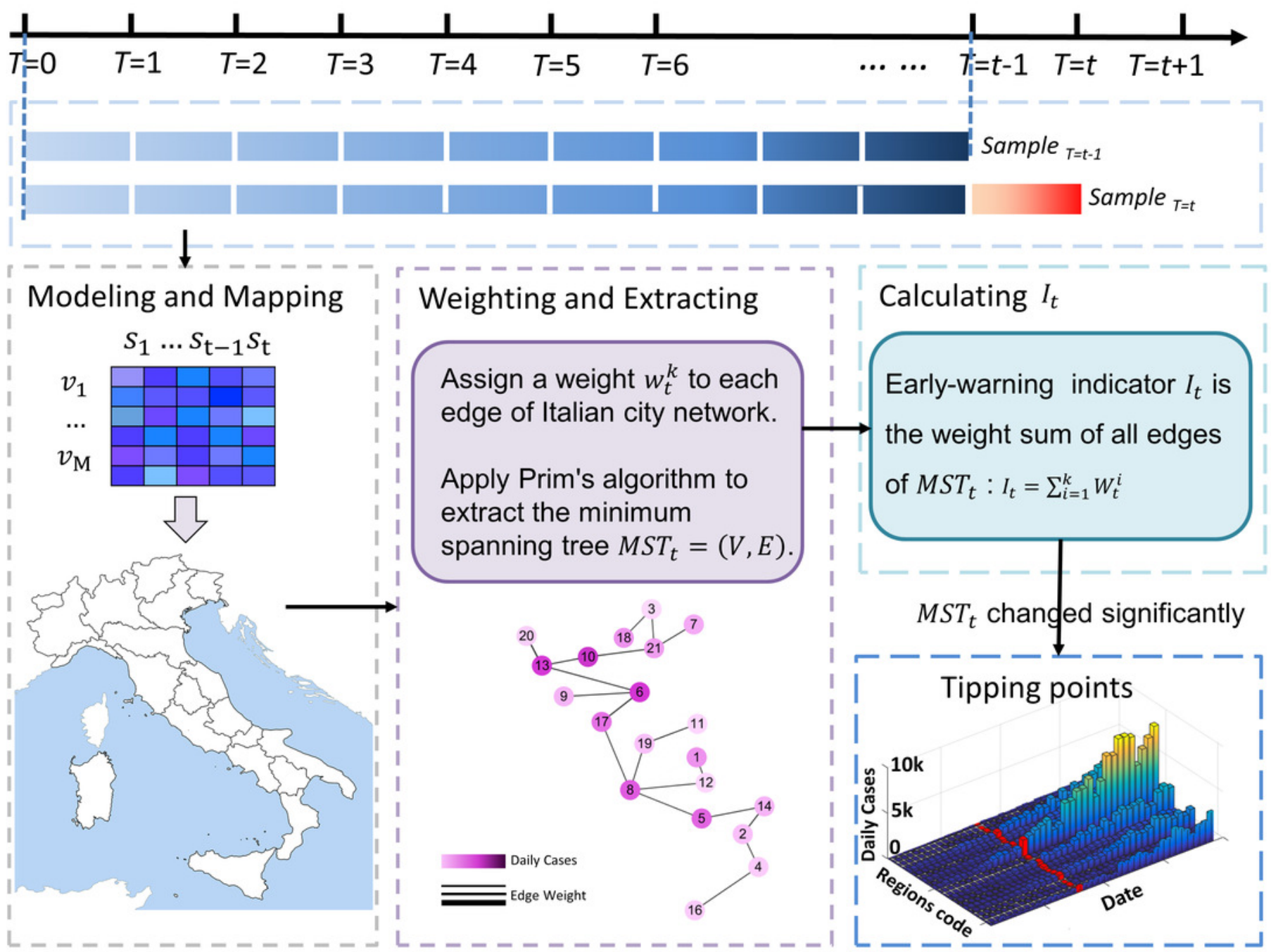


Figure 3

The region network model of Italy.

(A) A 21-node network model is constructed based on the geographic information and adjacent relationships of 21 regions/autonomous provinces in Italy. (B) A detailed list of correspondences between regions/autonomous provinces and nodes.

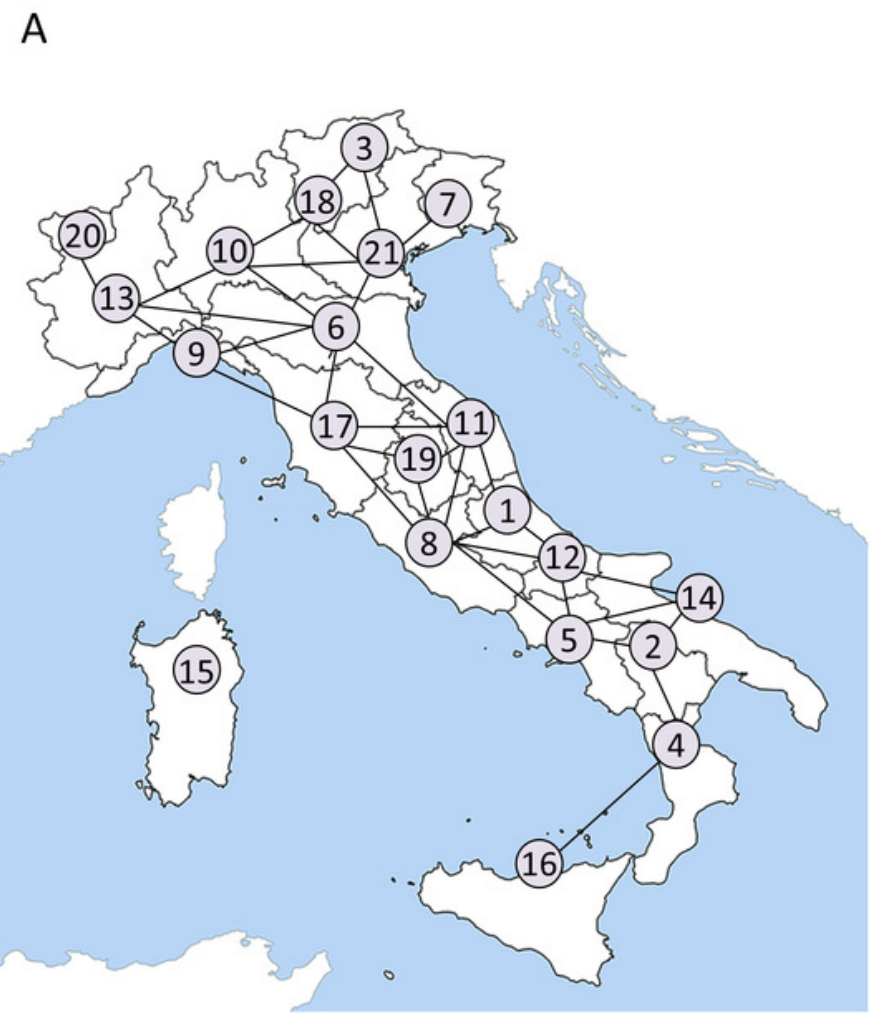

\begin{tabular}{|c|c|c|c|}
\hline B & \multicolumn{4}{|c|}{ Node } & $\begin{array}{c}\text { Region/Autono } \\
\text { mous province }\end{array}$ & Node & $\begin{array}{c}\text { Region/Autono } \\
\text { mous province }\end{array}$ \\
\hline 1 & Abruzzo & 11 & Marche \\
\hline 2 & Basilicata & 12 & Molise \\
\hline 3 & P.A. Bolzano & 13 & Piemonte \\
\hline 4 & Calabria & 14 & Puglia \\
\hline 5 & Campania & 15 & Sardegna \\
\hline 6 & Emilia Romagna & 16 & Sicilia \\
\hline 7 & Friuli Venezia & 17 & Toscana \\
\hline 8 & Giulia & 18 & P.A. Trento \\
\hline 9 & Lazio & 19 & Umbria \\
\hline 10 & Liguria & 20 & Valle d'Aosta \\
\hline
\end{tabular}


Figure 4

Forecast of the COVID-19 outbreaks in Italy.

In each subgraph, the left $y$-axis is the number of daily cases in Italy and the right $y$-axis is the MST-DNM indicator of corresponding date. (A) For the first wave of COVID-19 outbreak in Italy, the early-warning signal was about 15 days ahead of the outbreak point. (B) For the second wave of COVID-19 epidemic, the MST-DNM indicator significantly increases about 10 days before the actual number of confirmed cases skyrockets. 

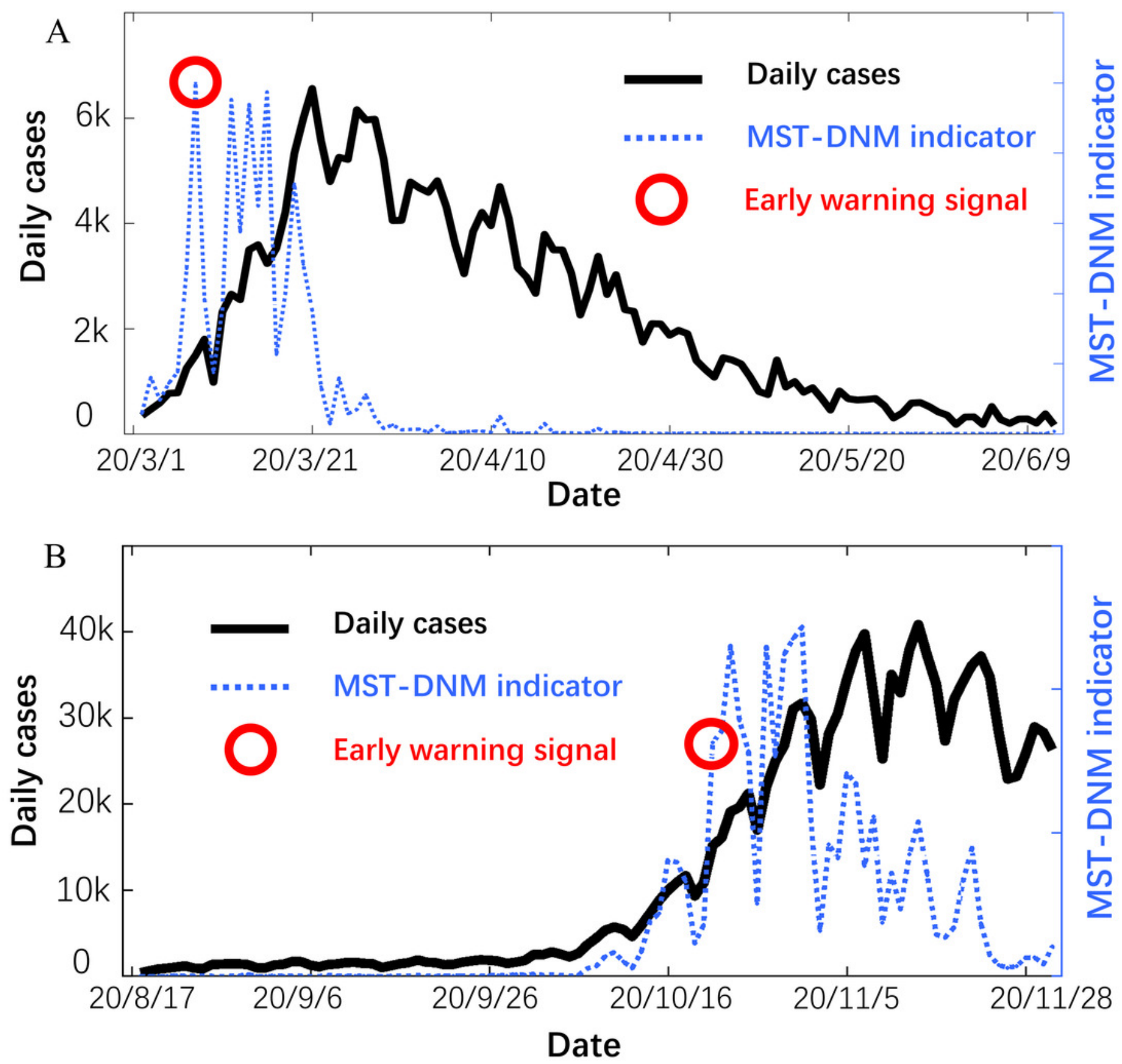


\section{Figure 5}

Dynamic evolution of the minimum spanning tree extracted from Italian region network.

The tree networks are respectively selected on September 26th (the normal stage), October 12th (the normal stage), October 20th (the pre-outbreak stage), October 26th (the preoutbreak stage), November 11th (the outbreak stage) and November 28th (the outbreak stage). In each subgraph, each node is colored by the number of daily cases with MinMaxScaler in each region and the thickness of the edge represents the correlation between two vertices of the edge. It is clear that the edges become thicker before the nodes turn darker, which indicates our model can identify the early-warning signal when the actual number of confirmed cases do not increase significantly. 


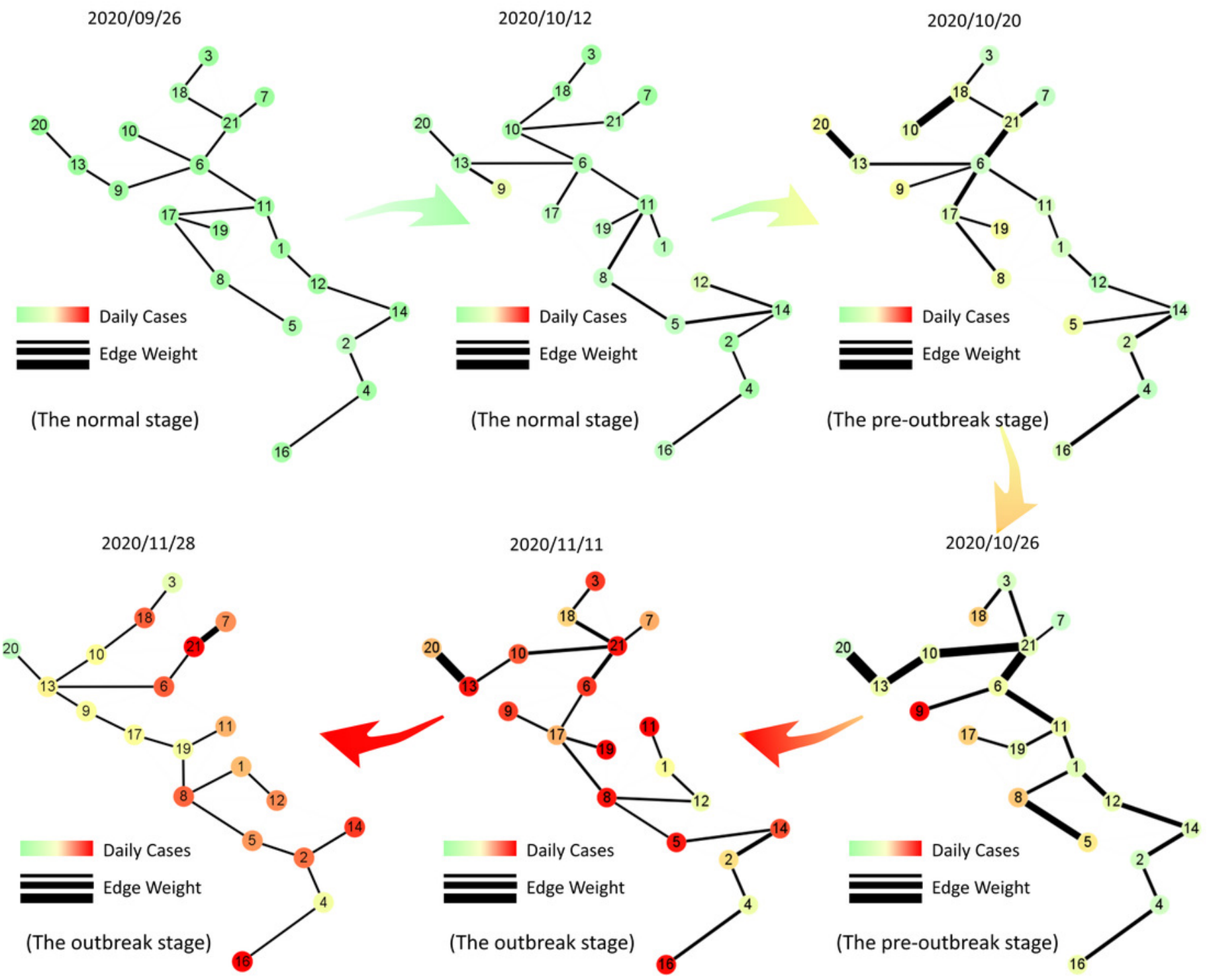




\section{Figure 6}

Demonstration of early warning function in Italy.

(A) The minimum spanning tree of Italian dynamic region network on October $26^{\text {th }}$, colored by the scaled value of daily cases and the weight of edges, is divided into two local networks according to the thickness of the edges. (B) The stacked bar chart of new cases in Italy shows the severity of the COVID-19 outbreak after the early warning, where the blue columns represent the cumulative number of confirmed cases in each regions/ autonomous provinces in Italy as of October 26, and the orange columns represent the total number of new confirmed cases from October 27 to November 28. In particular, the red regional names highlight the five regions with the most confirmed cases. In combination, it is clear that two high-risk regions are identified by the minimum spanning tree extracted from the dynamic region network. 
A The minimum spanning tree of Italian dynamic region network on October 26
B

Stacked bar chart of new cases in Italy as of November 28

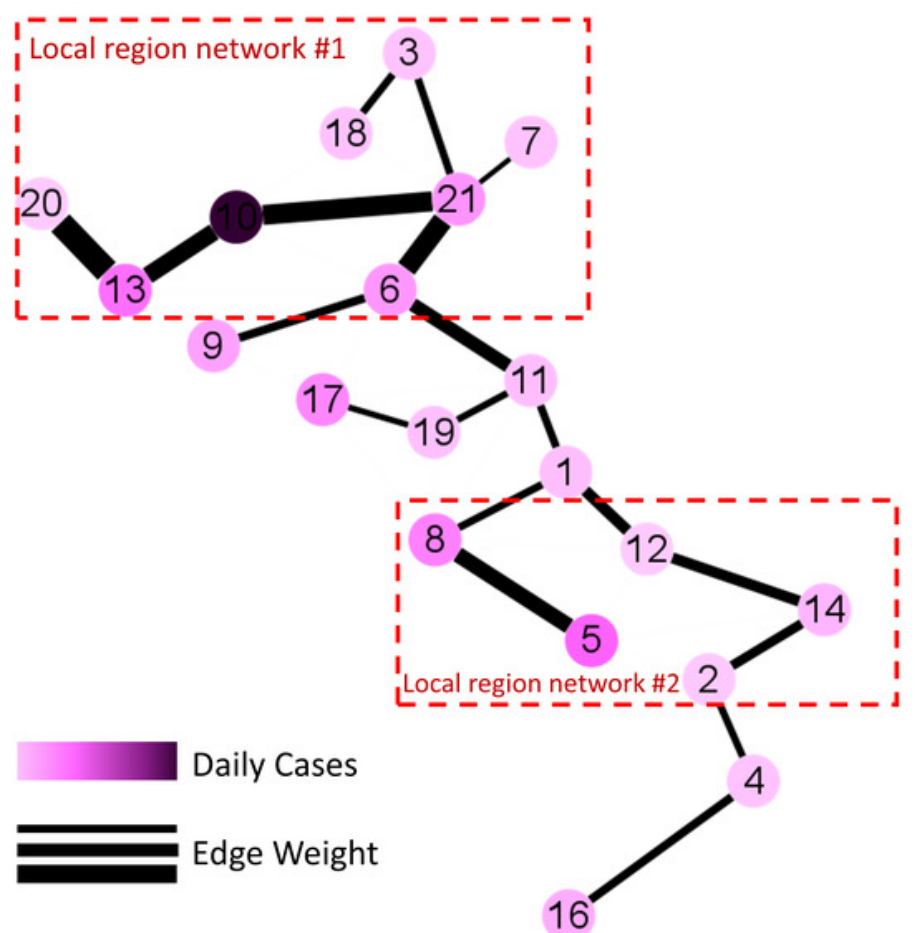

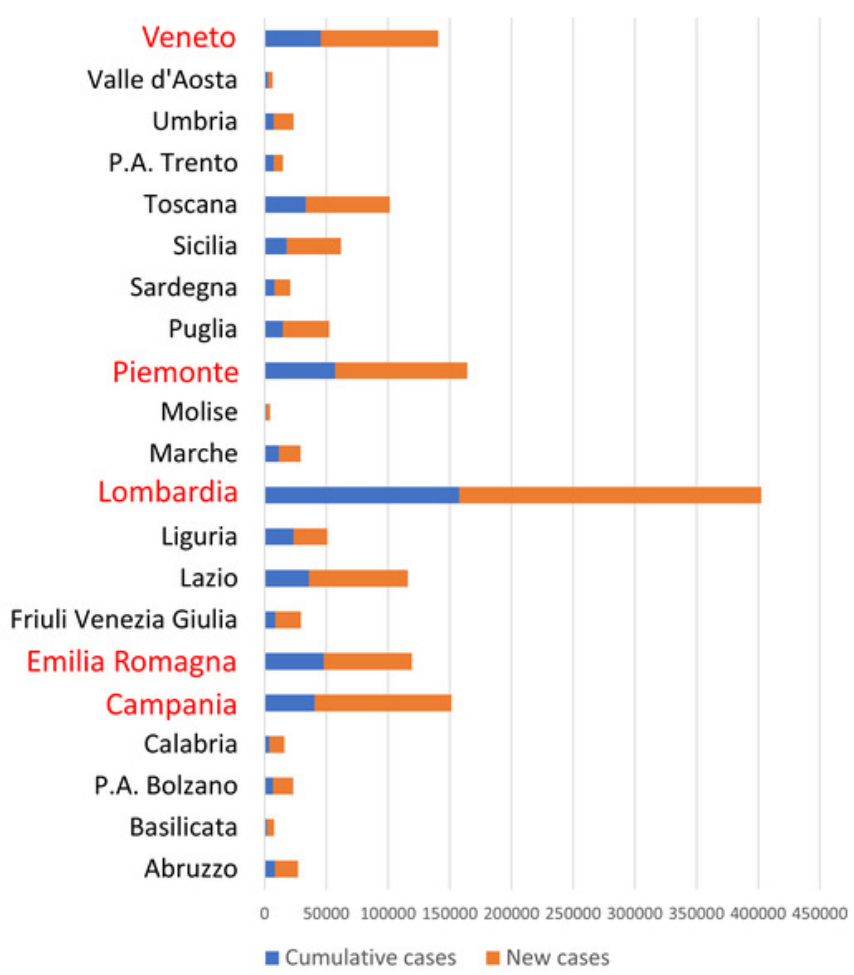


Table $\mathbf{1}$ (on next page)

Critical behaviours of the MST-DNM indicator $I_{t}$ for different cases 
1 Table 1 Critical behaviours of the MST-DNM indicator $I_{t}$ for different cases

\begin{tabular}{c|ccccccc}
\multicolumn{1}{c}{ CASE } & NODES & $S D_{t}$ & $\left|\Delta S D_{t}(i . j)\right|$ & $P C C_{t}(i, j)$ & $\left|\Delta P C C_{t}(i, j)\right|$ & $W_{t}^{k}$ & $I_{t}$ \\
\hline $\mathbf{1}$ & All DNM & $\nearrow$ & $\nearrow$ & $\nearrow$ & $\nearrow$ & $\nearrow$ & $\nearrow$ \\
\multirow{2}{*}{$\mathbf{2}$} & DNM and & $\mathrm{D} \nearrow$ & & $\mathrm{D} \nearrow$ & $\nearrow$ & & \\
& non-DNM & $\mathrm{N} \rightarrow$ & $\nearrow$ & $\mathrm{N} \backslash$ & $\nearrow$ & &
\end{tabular}

2

3 Notes: When the system moves from time point $t-1$ to $t$, it is approaching the critical point.

41 . “ $\nearrow$ " represents the increase of variables; " \" represents the decrease of variables; " $\rightarrow$ " represents that 5 there is no significant change in the variables;

62 2. "D" represents the DNM members; "N" represents the non-DNM members;

$73 . S D_{t}$ is the standard deviation at time point $t ; P C C_{t}(i, j)$ is the Pearson's correlation coefficient between 8 two nodes $v_{i}, v_{j}$. 
Table 2 (on next page)

Description of the early warning function of edge weight 
1 Table 2 Description of the early warning function of edge weight

\begin{tabular}{|c|c|c|c|c|}
\hline $\begin{array}{c}\text { Local region } \\
\text { network \# }\end{array}$ & Edge & Related regions & $\begin{array}{c}\text { Newly added } \\
\text { cases }\end{array}$ & Growth rate \\
\hline \multirow{6}{*}{1} & \multirow{2}{*}{$e\left(v_{10}, v_{21}\right)$} & Lombardy & 244726 & $154.96 \%$ \\
\hline & & Veneto & 95506 & $210.07 \%$ \\
\hline & \multirow[t]{2}{*}{$e\left(v_{6}, v_{21}\right)$} & $\begin{array}{l}\text { Emilia- } \\
\text { Romagna }\end{array}$ & 71379 & $148.93 \%$ \\
\hline & & Veneto & 95506 & $210.07 \%$ \\
\hline & \multirow{2}{*}{$e\left(v_{13}, v_{20}\right)$} & Piedmont & 107150 & $187.46 \%$ \\
\hline & & Valle d'Aosta & 3747 & $140.39 \%$ \\
\hline \multirow{4}{*}{2} & \multirow{2}{*}{$e\left(v_{5}, v_{8}\right)$} & Campania & 111077 & $273.91 \%$ \\
\hline & & Lazio & 80262 & $223.35 \%$ \\
\hline & \multirow{2}{*}{$e\left(v_{12}, v_{14}\right)$} & Molise & 3193 & $242.08 \%$ \\
\hline & & Puglia & 37341 & $249.39 \%$ \\
\hline
\end{tabular}

2 Notes:

3 1. "Newly added cases" refers to the cumulative number of confirmed cases in the corresponding region $4 \quad$ from October 26 to November 28.

5 2. "Growth rate" refers to the newly confirmed cases in corresponding regions from October 26 to $6 \quad$ November 28 divided by the cumulative cases on October 26. 


\section{Box 1(on next page)}

The indicator $I_{t}$ using Prim's algorithm 


\section{Algorithm}

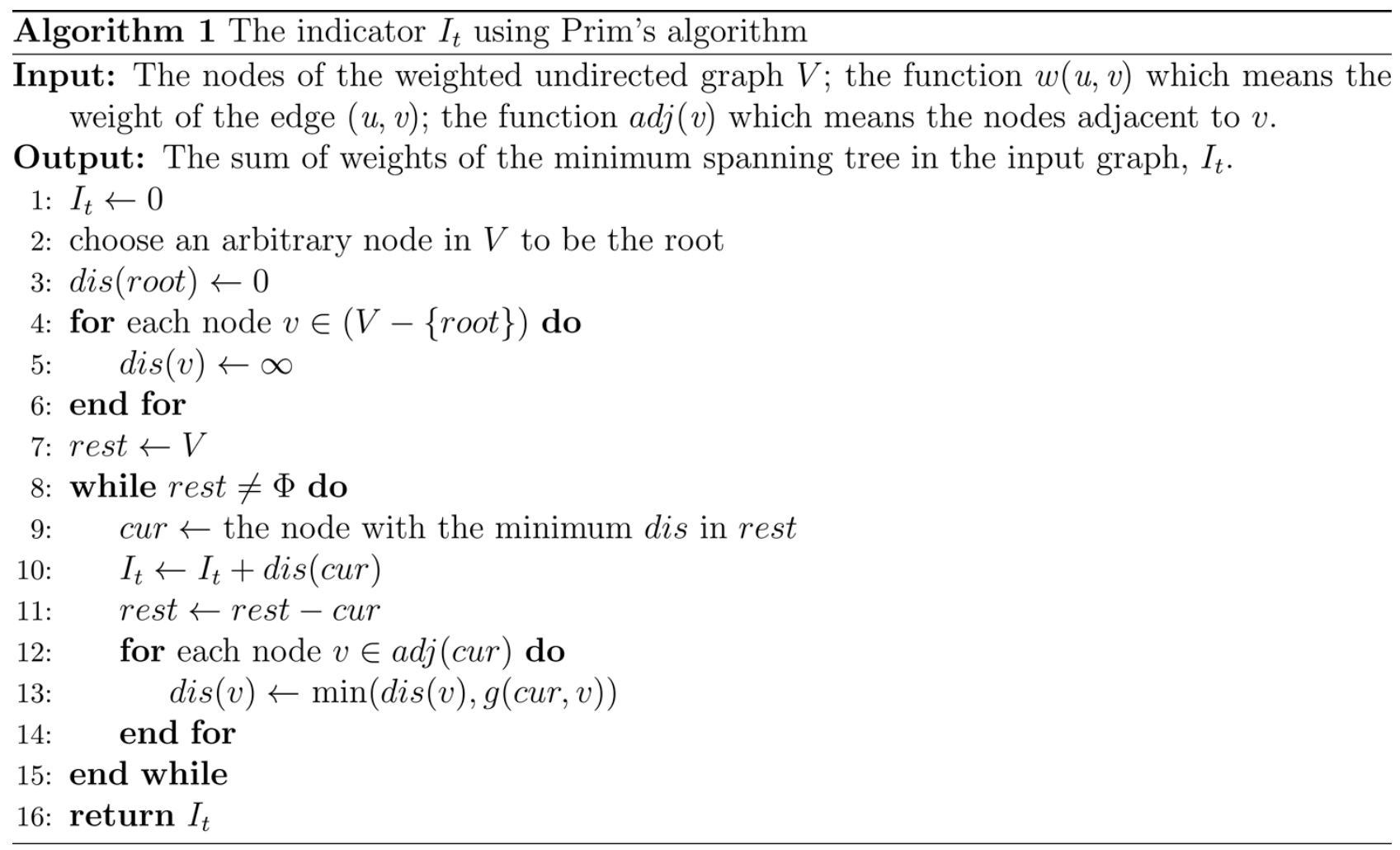

3

4

5

\section{LaTeX code:}

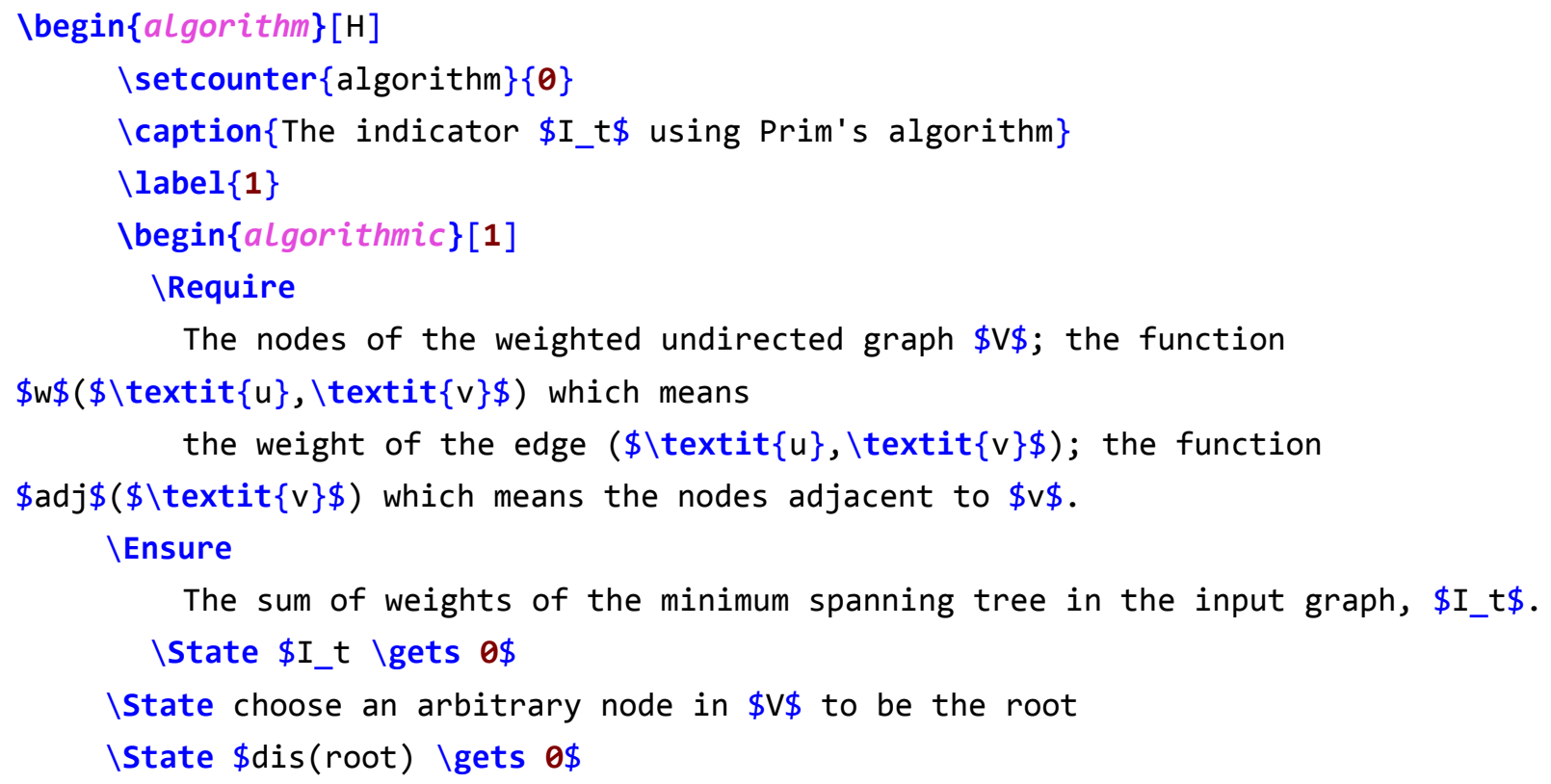




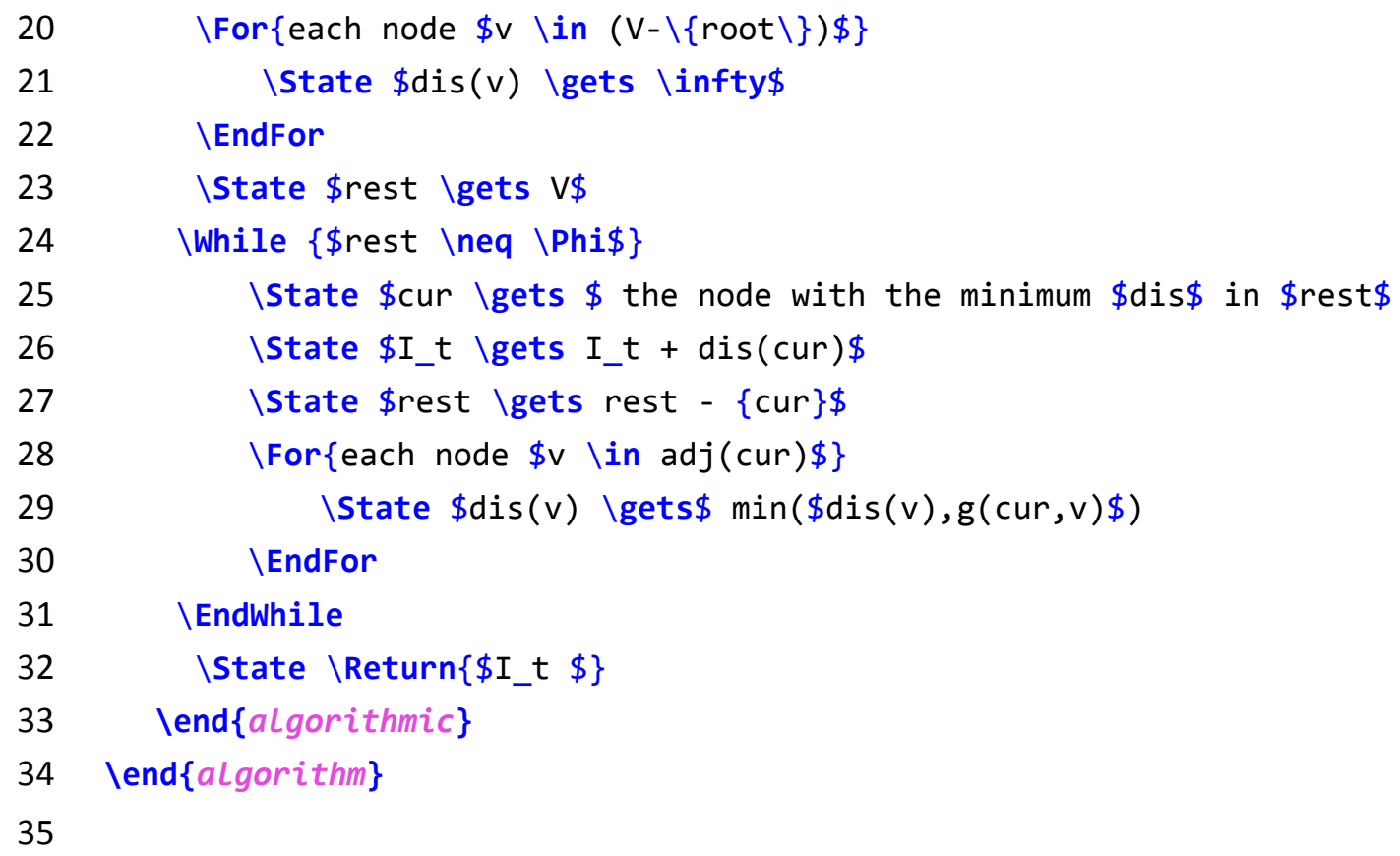

